\title{
Association of Pregestational BMI and Antenatal Weight Gain With Pregnancy Outcome: A Prospective Observational Cohort Study
}

\author{
Radhakanta Pal ${ }^{1}$, Mainak Maiti ${ }^{2}$, Bidisha Roychoudhury ${ }^{3 *}$, Poushali Sanyal ${ }^{3}$, Bijit Chowdhury $^{4}$
}

\begin{abstract}
Objectives: A prospective observational study to analyze whether optimal weight gain in pregnancy with respect to pregestational body mass index (BMI) can influence pregnancy outcome.

Materials and Methods: The study was done in the department of obstetrics and gynaecology at Ramakrishna Mission Seva Pratishthan, Vivekananda Institute of Medical Sciences, Kolkata over a period of one year. Two hundred early booked (before 10 weeks) singleton pregnant women were involved. According to pregestational BMI, and total antenatal weight gain all women were categorized into groups and the pregnancy outcome was compared in different groups. All the data were statistically analyzed using Chi-square test for categorical variables and analysis of variance (ANOVA) test for continuous variables using the SPSS version 16. Results: Analysis shows antenatal weight gain has a statistically significant $(P<0.001)$ relation with pregestational BMI with a significant increase in antenatal complications and caesarean section associated with obesity but not with the total antenatal weight gain. However the birth weight of the baby has a significant relation with total antenatal weight gain.

Conclusion: Our study concluded that most of the Indian women do not have weight gain as per Institute of Medicine (IOM) recommendation, with increased risk of medical and surgical complications in obese group of women.

Keywords: Pregestational BMI, Antenatal weight gain, Pregnancy outcome, Pregnancy complications, obesity
\end{abstract}

\section{Introduction}

Antenatal weight gain is of prime importance for successful outcome of mother and also for the baby. Maternal body mass index (BMI) and the weight that a mother gains during pregnancy are two of the strongest predictors of the birth weight and perinatal outcome (1-3). Various literatures have documented relationship between adverse pregnancy outcome and maternal obesity (4). Many studies have elaborated the fact that low maternal weight gain is a preventable risk factor for low birth weight babies.

In 1990, Institute of Medicine (IOM) (1) published a report showing association between pregnancy weight gain and infant size and provided target ranges of recommended weight gains by pre-pregnancy BMI. In 1995 World Health Organization Collaborative Study on Maternal Anthropometry and Pregnancy Outcome reviewed information from different countries to define desirable maternal weight gain.

This study determines the usefulness and effectiveness of this recommendation in our population and to compare outcomes of pregnancy in different weight gain groups. This study may be helpful for the expecting mother for more consistent and evidence based recommendations for desirable pregestational and gestational nutritional status and expected weight gain during pregnancy for better outcome.

\section{Materials and Methods}

The study was done in the department of obstetrics and gynaecology at Ramakrishna Mission Seva Pratishthan, Vivekananda Institute of Medical Sciences, Kolkata over a period of one year after approval was given by the hospital ethical committee dated 21st December 2010. Two hundred early booked (before 10 weeks) singleton pregnancy without known medical complications like chronic hypertension, diabetes, were included in the study. In the first visit their height and weight were measured and BMI was calculated. According to BMI, they were divided into four groups: underweight $\left(<18.5 \mathrm{~kg} / \mathrm{m}^{2}\right)$, normal weight $\left(18.5-24.9 \mathrm{~kg} / \mathrm{m}^{2}\right)$, over weight $\left(25-29.9 \mathrm{~kg} / \mathrm{m}^{2}\right)$ and obese $\left(\geq 30 \mathrm{~kg} / \mathrm{m}^{2}\right)$. Total antenatal weight gain were calculated and all the patients were divided in three groups (low, normal, high pregnancy weight gain) as per IOM recommendation (1). Different complications during antenatal peri-

Received 3 March 2016, Accepted 27 October 2016, Available online 1 January 2017

${ }^{1}$ Department of Gynecological Oncology, Tata Medical Center, Kolkata, West Bengal, India. ${ }^{2}$ Department of Obstetrics and Gynecology, KPC Medical College and Hospital, Jadavpur, Kolkata, West Bengal, India. ${ }^{3}$ Department of Obstetrics and Gynecology, Institute of Postgraduate Medical Education \& Research, Kolkata, West Bengal, India. ${ }^{4}$ Department of Obstetrics and Gynecology, Vivekananda Institute Of Medical Sciences, Ramakrishna Mission Seva Pratishthan, Kolkata, West Bengal, India.

*Corresponding Author: Dr. Bidisha Roychoudhury; Address: 54/B, Raja Rammohan Sarani, Kolkata - 700009, India. Tel: +91 9831645879; Email: bidisha_roychoudhury@yahoo.co.in 
ods e.g., gestational diabetes mellitus (GDM), gestational hypertension, mode of delivery, post-operative complication, neonatal complication were recorded during hospital stay and in the postnatal period. Then the pregnancy outcomes were compared in different gestational weight gain groups.

\section{Results}

Total 200 patients have been studied as per inclusion criteria. They were divided into different BMI groups and three weight gain groups i.e. low, normal and high weight gain groups. All the data were statistically analyzed by SPSS version 16 using chi-square test for categorical variables and analysis of variance (ANOVA) test for continuous variables and $P$ value less than 0.01 has been taken as highly significant. The analysis shows no statistical relation of antenatal weight gain with parity, occupation, religion, residence, education and diet of women although the incidence of low weight gain was $64.2 \%$ among under graduates whereas $36.4 \%$ among postgraduates and the incidence of low weight gain was $87.5 \%$ among vegetarians whereas $57.8 \%$ among non-vegetarians. However results showed that mothers who took vegetarian diet have high incidence of low birth weight and it was statistically significant $(P<0.001)$. Table 1 shows distribution of antenatal weight gain in different BMI group. It has a statistically significant relationship with pregestational BMI $(P<0.001)$. The analysis of antenatal complications in the different antenatal weight gain groups showed that the incidence of GDM, gestational hypertension, preterm births were not significant. However Table 2 shows development of GDM is significantly related with pregestational BMI $(P<0.001)$; Table 3 shows development of gestational hypertension is more common in obese women and it was statistically significant $(P=0.026)$. Analysis of the intrapartum period showed that the gestational age at delivery in the different antenatal weight gain and BMI groups were not significant, although the BMI has a significant relation $(P=0.023)$, with the mode of delivery showing obese mothers having high incidence of caesarean section. In the postpartum period analysis showed high incidence of wound infection among high weight gain group and it was statistically significant $(P=0.016)$. While analyzing the neonatal outcome it was seen that the different antenatal weight gain groups and BMI groups did not have any statistical relationship with the number of NICU admissions or the Apgar score taken at 1 and 5 minutes of the babies, however the birth weight of babies in Table 4 shows that there was significant relation $(P=0.004)$ between antenatal weight gain and birth weight.

\section{Discussion}

Our study shows antenatal weight gain has a role in respect to fetal and maternal outcome. The incidence of low weight gain is high (64.2\%) among mothers who are under graduate than those mothers who were post graduate (36.4\%), but statistically, it was not significant. Unlike the study by Li et al (5) pregestational obese mothers were older, belonged to lower socioeconomic group compared with mothers with pregestational normal weight. In the review article by Muthayya (6) in the developing regions of the world lack of resources and education are determining factors for the health outcome of mothers and babies. Thus it showed a lower incidence of IUGR with higher level of maternal education ranging from $46 \%$ in women who had no schooling to $19 \%$ in women who had a post-graduate education According to our study, obese and overweight patients have higher propensity to gain more weight than normal value BMI patients and consequently have adverse pregnancy outcome. Moreover, those who have low BMI also have more risk of low weight gain and poor pregnancy outcome. BMI is significantly $(P<0.001)$ related to maternal weight gain during pregnancy. Antenatal weight gain is much more with high BMI. Like in the study by Ee et al (7) in 2014 showed antenatal weight gain among Asian women who are obese, should be lower. However, the optimal weight gain for underweight and obese women was outside the IOM recommended range.

Table 1. Relationship of Pregestational BMI With Antenatal Weight Gain

\begin{tabular}{lcccccc}
\hline \multirow{2}{*}{$\begin{array}{l}\text { Antenatal Weight } \\
\text { Gain }\end{array}$} & \multicolumn{2}{c}{ BMI } & \multirow{2}{*}{ Total } & \multirow{2}{*}{$\boldsymbol{P}$ Value } \\
\cline { 2 - 5 } & Under Weight & Normal & Over Weight & Obese & & \\
\hline Low weight gain & $12(92.3)$ & $79(75.2)$ & $25(38.5)$ & $2(11.8)$ & $118(59)$ & $<0.001^{*}$ \\
Normal weight gain & $1(7.7)$ & $22(21)$ & $29(44.6)$ & $7(41.2)$ & $59(29.5)$ & $<0.001^{*}$ \\
High weight gain & $0(0)$ & $4(3.8)$ & $11(16.9)$ & $8(47.1)$ & $23(11.5)$ & $<0.001^{*}$ \\
Total & $13(100)$ & $105(100)$ & $65(100)$ & $17(100)$ & $200(100)$ & \\
\hline
\end{tabular}

*Significant.

Table 2. Relation of Pregestational BMI With Development of GDM

\begin{tabular}{lcccccc}
\hline \multirow{2}{*}{ GDM } & \multicolumn{3}{c}{ BMI } & \multirow{2}{*}{ Total } & \multirow{2}{*}{$\boldsymbol{P}$ Value } \\
\cline { 2 - 5 } & Under Weight & Normal & Over Weight & Obese & & \\
\hline No & $13(100)$ & $95(90.5)$ & $52(80)$ & $8(47.1)$ & $168(84)$ & $<0.001^{*}$ \\
Yes & $0(0)$ & $10(9.5)$ & $13(20)$ & $9(52.9)$ & $32(16)$ & \\
Total & $13(100)$ & $105(100)$ & $65(100)$ & $17(100)$ & $200(100)$ & \\
\hline
\end{tabular}

*Significant. 
Table 3. Relation of Pregestational BMI With Development of Gestational HTN

\begin{tabular}{lcccccc}
\hline \multirow{2}{*}{$\begin{array}{l}\text { Gestational } \\
\text { HTN }\end{array}$} & \multicolumn{3}{c}{ BMI } & \multirow{2}{*}{ Total } & \multirow{2}{*}{$\boldsymbol{P}$ Value } \\
\cline { 2 - 5 } & Under Weight & Normal & Over Weight & Obese & & \\
\hline No & $13(100)$ & $92(87.6)$ & $61(93.8)$ & $12(70.6)$ & $178(89)$ & $0.026^{*}$ \\
Yes & $0(0)$ & $13(12.4)$ & $4(6.2)$ & $5(29.4)$ & $22(11)$ & \\
Total & $13(100)$ & $105(100)$ & $65(100)$ & $17(100)$ & $200(100)$ \\
\hline
\end{tabular}

*Significant.

Table 4. Relationship of Birthweight of Babies With Antenatal Weight Gain of Mothers

\begin{tabular}{lcccc}
\hline \multirow{2}{*}{$\begin{array}{l}\text { Antenatal Weight } \\
\text { Gain }\end{array}$} & \multicolumn{3}{c}{ Birthweight } & \multicolumn{1}{c}{ Total } \\
\cline { 2 - 4 } & $30(85.7)$ & $88(53.7)$ & High & $118(59)$ \\
Low weight gain & $5(14.3)$ & $53(32.3)$ & $1(100)$ & $0.004 *$ \\
Normal weight gain & $0(0)$ & $23(14)$ & $0(0)$ & $0.004 *$ \\
High weight gain & $35(100)$ & $164(100)$ & $1(100)$ & $29.5)$ \\
Total & & & & $200(11.5)$ \\
\hline
\end{tabular}

*Significant.

Chang et al (8) in their study has shown almost similar result in 2010. Our study shows that incidence of GDM is double in high weight gain group (26.1\%) than low weight gain group (13.6\%). High weight gain also increases the chance of development of gestational hypertension $(6.8 \%$ in normal weight gain mother and $21.7 \%$ in high weight gain mothers). There was no significant $(P=0.058)$ relationship between weight gain during the pregnancy and gestational age or mode of delivery. But it is significantly related to BMI of the patient $(82.4 \%$ caesarean section rate in obese patients and 55.2\% in normal BMI patients). Hedderson et al (9) in 2010, Yin et al (10) and Yekta et al (11) have shown similar results in 2005. The study by Haugen et al (12) in 2014 showed significantly increased risk of pregnancy induced hypertension, macrosomia, and emergency cesarean delivery in both nulliparous and parous normal weight women and overweight women except for no increased risk for gestational hypertension in parous women with antenatal weight gain more than the IOM recommendation. Liu et al (13) showed that compared with antenatal weight gain within the IOM recommendations, excessive weight gain increased the incidence of cesarean section, preterm delivery, preeclampsia and infant macrosomia, and reduced the incidence of GDM, while inadequate antenatal weight gain increased the incidence of GDM and SGA. In our study, there is no significant $(P=0.226)$ relation between antenatal weight gain and preterm delivery, though statistically not significant, preterm birth is much more prevalent in the low weight gain group $(10.2 \%)$ than high weight gain group (4.3\%). Radhakrishnan et al (14) in 2013 showed preterm deliveries were associated with less than optimal weight gain (adjusted odds ratio [OR] 3.58, 95\% CI: 1.757.32) after adjusting for gestational age at delivery. Similarly in the study by Tabatabaei (15) in Iran the ORs for preterm delivery were significantly less in the groups with a pregestational BMI greater than normal. In our study weight gain during pregnancy is directly proportional to the birth weight of the baby. Low weight gain significantly
$(P<0.004)$ increases the incidence of low birth weight. In 2010 Chang et al (8) in Taiwan and Haugen et al (12) in 2014 proved weight gain less than the IOM recommendations increased the risk of low birth weight baby among normal weight nulliparous women. Our study shows no significant correlation between antenatal weight gain and NICU admission $(P=0.585)$ of the baby. There is also no significant relation between weight gain during pregnancy and 1 minute $(P=0.876)$ and 5 minutes $(P=0.4)$ Apgar score. Though Choi et al (16) in their study had shown that there is inverse relationship between low Apgar score and NICU admission with antenatal weight gain. Unlike the study in 2013 by Radhakrishnan et al (14) antenatal weight gain was not associated with neonatal outcomes in their study population of south Indian women.

\section{Conclusion}

In our study we found that only $29.5 \%$ of the patients have weight gain within normal range as described by the IOM (1). We found that increase antenatal weight gain also increases the risk of developing GDM, hypertension, wound infection. Low weight gain group has also increase chance of pre-term delivery and low birth weight baby. Antenatal weight gain thus is a very important issue to pregnant woman as well as her physician. Time to time different recommendations has been made to optimize weight gain as it has been shown by different studies that optimal weight gain can influence pregnancy outcome.

\section{Ethical Issues}

Informed consent was taken from all of them, the format of which was approved by the hospital ethical committee.

\section{Conflict of Interests}

None of the authors had conflict of interests with any issues.

\section{Financial Support}

This study was supported by Dean and Secretary Maharaj, 
of Ramakrishna Mission Seva Pratishthan hospital, VIMS, Kolkata, India.

\section{Acknowledgments}

We would like to thank Mr. Souvik Dutta for statistical analysis and our patients who had shown their faith in us.

\section{References}

1. Weight Gain During Pregnancy IOM. Reexamining the guidelines. Washington, DC: National Academies Press; 2009:370.

2. Bodnar LM, Catov JM, Klebanoff MA, Ness RB, Roberts JM. Prepregnancy body mass index and the occurrence of severe hypertensive disorders of pregnancy. Epidemiology. 2007;18:234-239. doi:10.1097/01.ede.0000254119.99660. e7.

3. Cedergren M. Effects of gestational weight gain and body mass index on obstetric outcome in Sweden. Int J Gynaecol Obstet. 2006;93(3):269-274. doi:10.1016/j.ijgo.2006.03.002.

4. Ehrenberg H, Dierker L, Milluzzi C, Mercer B. Low maternal weight, failure to thrive in pregnancy, and adverse pregnancy outcomes. Am J Obstet Gynecol. 2003;189:17261730. doi:10.1016/j.ajog.2004.05.052.

5. Li N, Liu E, Guo J. Maternal prepregnancy body mass index and gestational weight gain on pregnancy outcomes. PLoS One. 2013;8(12):e82310. doi:10.1371/journal. pone.0082310.

6. Muthayya S. Maternal nutrition \& low birth weight - what is really important? Indian J Med Res. 2009;130:600-608.

7. Ee TX, Allen JC, Malhotra R, Koh H, Østbye T, Tan TC. Determining optimal gestational weight gain in a multiethnic Asian population. J Obstet Gynaecol Res. 2014;40:1002-1008. doi:10.1111/jog.12307

8. Chang MU, Kuo CH, Chiang KF. The effects of prepregnancy body mass index and gestational weight gain on neonatal birth weight in Taiwan. Int J Nurs Midwifery.
2010;2(2):28-34.

9. Hedderson MM, Gunderson EP, Ferrara A. Gestational weight gain and risk of gestational diabetes mellitus. Obstet Gynecol. 2010;115(3):597-604. doi: 10.1097/ AOG.0b013e3181cfce4f

10. Yin YZ, Chen XW, Li XM, Hou HY, Zhou SS. Relations of pre-pregnant weight and weight gain during pregnancy with pregnancy-induced hypertension and birth weight. Di Yi Jun Yi Da Xue Xue Bao. 2005;25(2):226-8.

11. Yekta Z, Porali R, Aiatollahi H. The effect of pre-pregnancy body mass index, gestational weight gain on pregnancy outcomes. BMC Pregnancy Childbirth. 2006;6:15. doi: 10.1186/1471-2393-6-15.

12. Haugen M, Brantsæter AL, Winkvist A, et al. Associations of pre-pregnancy body mass index and gestational weight gain with pregnancy outcome and postpartum weight retention:a prospective observational cohort study. BMC Pregnancy Childbirth. 2014;14:201. doi:10.1186/14712393-14-201.

13. Liu L, Hong Z, Zhang L. Associations of prepregnancy body mass index and gestational weight gain with pregnancy outcomes in nulliparous women delivering single live babies. Sci Rep. 2015;5:12863. doi:10.1038/srep12863.

14. Radhakrishnan U, Kolar G, Nirmalan PK. Cross-sectional study of gestational weight gain and perinatal outcomes in pregnant women at a tertiary care center in southern India. J Obstet Gynaecol Res. 2014;40:25-31. doi:10.1111/ jog.12115.

15. Tabatabaei M. Gestational weight gain, prepregnancy body mass index related to pregnancy outcomes in Kazerun, Fars, Iran. Journal of Prenatal Medicine. 2011;5(2):35-40.

16. Choi SK, Park IY, Shin JC. The effects of pre-pregnancy body mass index and gestational weight gain on perinatal outcomes in Korean women: a retrospective cohort study. Reprod Biol Endocrinol. 2011;9:6. doi:10.1186/1477-78279-6.

Copyright ( 2017 The Author(s); This is an open-access article distributed under the terms of the Creative Commons Attribution License (http://creativecommons.org/licenses/by/4.0), which permits unrestricted use, distribution, and reproduction in any medium, provided the original work is properly cited. 